\title{
Multi-wavelength observations of a flare spray and CME of 1 October 2001
}

\author{
Hui Li, Jianqi You, Xingfeng Yu, and Qindi Wu \\ Purple Mountain Observatory, Chinese Academy of Sciences, Nanjing 210008, PR China \\ Received 22 February 2002 / Accepted 22 May 2002
}

\begin{abstract}
Using the Multi-channel Infrared Solar Spectrograph (MISS) at the Purple Mountain Observatory (PMO), we obtained the $\mathrm{H} \alpha$, Ca II $8542 \AA$, He $10830 \AA$ spectra and slit-jaw $\mathrm{H} \alpha$ images of a large dramatic flare spray near the southwest solar limb on 1 October 2001. For this event, we also got Extreme-Ultraviolet (EUV) images at $171 \AA$ from TRACE and $195 \AA$ from EIT/SOHO, coronal images from the C2 and C3/LASCO coronagraph on SOHO, soft X-ray (SXR) images from the Soft X-ray Telescope (SXT) and hard X-ray (HXR) flux from the Hard X-ray Telescope (HXT) onboard Yohkoh, Xray flux from GOES, and $\mathrm{H} \alpha$ images and radio-frequency spectrograms from the National Astronomical Observatories of China (NAOC). The complex eruption event lasted about $40 \mathrm{~min}$ and was composed of two flaring and three distinct mass ejecting processes. The preliminary analysis leads to the following results: (1) the three peak-times on the HXR light curves correspond to the interactions and reconnections of coronal loops shown on the SXR images and are consistent with the flaring times in $\mathrm{H} \alpha$; (2) the first mass ejection process of this event is apparently related to the loop interaction and magnetic opening and reconnection, while the second mass ejection, i.e., the large flare spray, is associated with the second flare. There are bright fine arcs connecting the onset location of the spray and the footpoints of the flare loops. The rising speeds the three ejections in the sky plane are all $296 \mathrm{~km} \mathrm{~s}^{-1}$ and uniform in the early phase; (3) comparisons show that the flare spray has nearly the same shape in $\mathrm{H} \alpha, 171 \AA, 195 \AA$ and SXR images, which implies that plasma with temperatures differs by a factor of more than 100 can exist in the same spray and move by about $2 \times 10^{5} \mathrm{~km}$ with the same shape; (4) we infer from the height-time curves of the spray and CME that the observed CME by LASCO is closely related to the flare spray. However, we could not conclude what the trigger of the CME is due to the lack of more direct and convincing evidence, such as images from the LASCO C1 coronagraph. Meanwhile, the CME is accelerated at about $2 R_{\odot}$ presumably due to change in the large-scale magnetic field structure; (5) in the initial phase of the spray, all the $\mathrm{H} \alpha$, Ca II $8542 \AA$ and $\mathrm{He}_{\text {I }} 10830 \AA$ spectra show unusually broadened profiles and large blue-shift velocities, and the maximum of the line-of-sight velocity reaches $286 \mathrm{~km} \mathrm{~s}^{-1}$.
\end{abstract}

Key words. Sun: flares - Sun: infrared - Sun: corona

\section{Introduction}

The properties of flare spray have been clearly described by Warwick (1957). Early space observations have shown that both sprays and eruptive prominences are associated with coronal transient phenomena (white-light changes and type II/IV radio bursts) (Valniček 1964). The average velocity of flare spray is about $60 \mathrm{~km} \mathrm{~s}^{-1}$ at the minimum phase and about $450 \mathrm{~km} \mathrm{~s}^{-1}$ at the maximum phase (Cox 1999). Due to the large velocity and abrupt onset of flare spray, it can barely be observed with a narrow passband filter. Meanwhile, one may obtain a distorted shape of flare spray due to the limitation of the filter passband, which is often mistaken as a surge. Therefore, only a few flare sprays with complete observation data had been observed in the past decades. TandbergHanssen et al. (1980) tabulated the completely observed flare sprays with a full set of data from 1969 to 1974 and a total

Send offprint requests to: $\mathrm{Hui} \mathrm{Li}$,

e-mail: lihui@mail.pmo.ac.cn number of thirteen is given. In recent years, flare spray is found to be intimately related to coronal mass ejection (CME) and has received more and more attention from academic circles. Because of the improvement of observation techniques, e.g., the EUV instrument with high spatial resolution (Handy et al. 1999) and the wide passband filter based on coronagraphs (Webb \& Jackson 1981), we can acquire data of flare sprays with higher speed and spatial resolution (Gallagher et al. 2000; Harrison et al. 2001). The observational data of flare sprays are still limited so far, for example, the spectral data about flare sprays are rare and mostly from $\mathrm{H} \alpha$ (Öhman et al. 1967; Webb \& Jackson 1981) and $\mathrm{H} \beta$ (Maeda et al. 1978) observation. The Coronal Diagnostic Spectrometer (CDS) (Harrison et al. 1995) onboard SOHO can obtain EUV spectrograms in several lines, but the temporal resolution is rather low (Harrison et al. 2001). Meanwhile, the relation between flare spray and CME is uncertain and needs to be verified and confirmed by more observations. 


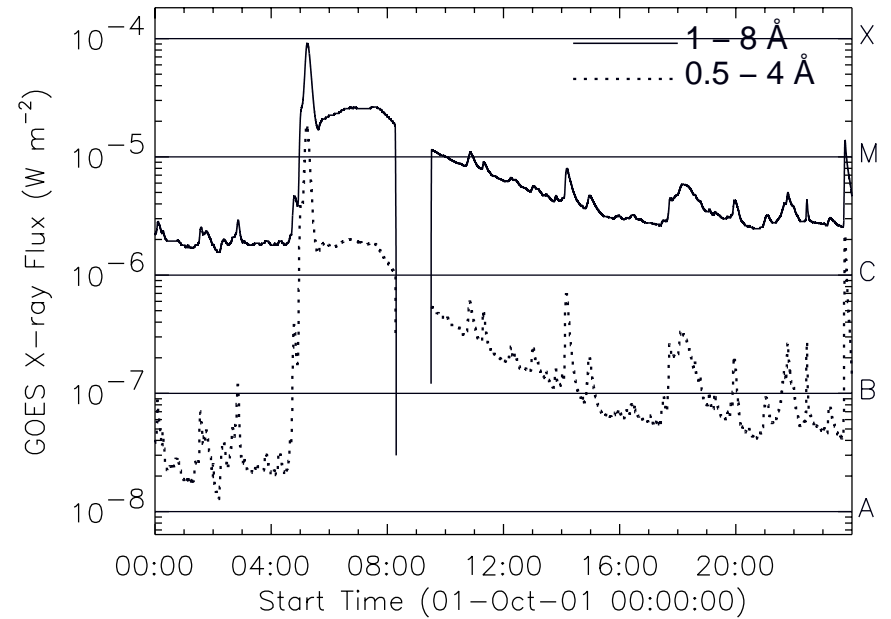

Fig. 1. GOES-10 X-ray flux of 1 October 2001.

We observed a large flare spray on 1 October 2001 and obtained complete set of data for this event (Table 1, and see next section for detail). This event provides a good example to understand the relationship between the behaviors of flare and spray in visible light, EUV, SXR and HXR. It is also suitable to address the physical status of flare spray and the relation between flare spray and CME. We will describe the observation and data in Sect. 2 and analyze the observed data in Sect. 3. The discussions and conclusions will be presented in Sect. 4.

\section{Observations}

There were two flare-productive $\delta$ active regions, AR 9628 and AR 9632, on the solar disk in the late September 2001. The center of AR 9628 was located on S17W79 at 05:00 UT of 1 October 2001 while its preceding sunspots were near the south-west solar limb. We monitored this active region with the Multi-channel Infrared Solar Spectrograph (MISS) (Li et al. 1999; Li et al. 2002) in order to observe solar limb flares. The MISS now uses three AP7p grade 1 scientific CCDs from Apogee Instrument instead of Pulnix TM-860 CCDs as its detectors and can observe the Sun simultaneously at three lines, i.e., $\mathrm{H} \alpha$, Ca II $8542 \AA$ and He I $10830 \AA$. The corresponding dispersions and exposure times for disk observation are $0.0545 \AA /$ pixel, $0.0511 \AA /$ pixel and $0.0478 \AA /$ pixel and $0.05 \mathrm{~s}$, $0.06 \mathrm{~s}$ and $0.4 \mathrm{~s}$, respectively (Li et al. 2002). The slit-jaw $\mathrm{H} \alpha$ image was taken by a Mintron MTV-1881EX CCD and an image grabber.

At about 04:40 UT on 1 October 2001, we found that there was an indication of prominence eruption and thus we observed this region. The $\mathrm{H} \alpha$, Ca II $8542 \AA$ and He I $10830 \AA$ spectrograms were taken by the MISS at a rate of about $2.8 \mathrm{~s}$ per frame, and the slit-jaw $\mathrm{H} \alpha$ images at a rate of about $30 \mathrm{~s}$ per frame. The observation lasted about $50 \mathrm{~min}$ and ended after the end of the large eruption. The Solar Geophysical Data (SGD) indicated that this is an M9.1 event (Fig. 1), but there is no registration of an $\mathrm{H} \alpha$ flare. However, our $\mathrm{H} \alpha$ observations (images and spectra) show that this spray was indeed accompanied by two short-lived small flares occurred at two different locations of AR 9628 (see next section for detail). We made
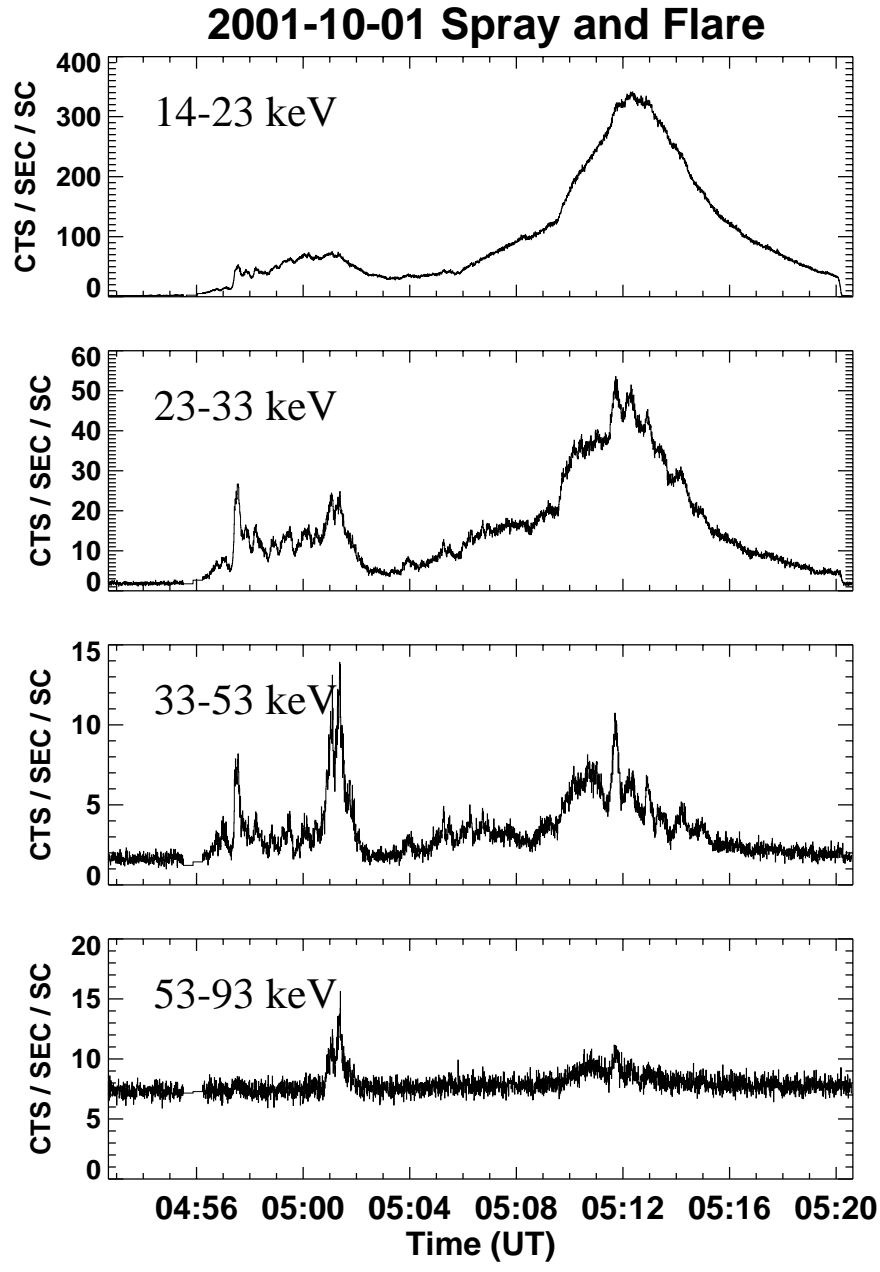

Fig. 2. HXR light curves of the four energy channels of HXT/Yohkoh on 1 October 2001.

corrections of the observed data for flat-field and dark current, and conducted intensity calibration. Figure 1 shows that the first peak of GOES X-ray flux appears at 04:45 UT and 04:48 UT for the 0.5-4 A and 1-8 A wavebands while the main peak at 05:12 UT and 05:15 UT, respectively. The main peak of 0.5-4 $\AA$ is consistent with the main HXR peak (Fig. 2) while that of 1-8 $\AA$ peaked 3 min later.

We also got other data for this event. The data, in addition to what mentioned above, include $\mathrm{H} \alpha$ images from Huairou Solar Observing Station (HSOS) of NAOC, EUV images at $171 \AA$ from the Transient Region and Coronal Explorer (TRACE) (Handy et al. 1999) and $195 \AA$ from the Extreme-ultraviolet Imaging Telescope (EIT) (Delaboudinière et al. 1995) onboard SOHO, coronal images from C2 and C3 of the Large Angle Spectroscopic Coronagraph (LASCO) (Brueckner et al. 1995) on SOHO, SXR images and HXR flux from SXT (Tsuneta et al. 1991) and HXT (Kosugi et al. 1991) onboard Yohkoh, X-ray flux from GOES-10 satellite, and radio-frequency spectrograms from NAOC.

\section{Data and analysis}

We now analyze the observed data. This complicated eruption event includes three distinct erupting and two flaring 
Table 1. List of observation times and average sampling rates.

\begin{tabular}{|c|c|c|}
\hline Observation type & time period (UT) & average sampling rate \\
\hline Spectroscopic obs. .. & $04: 58-05: 50$ & $2.8 \mathrm{~s}$ \\
\hline Slit-jaw $\mathrm{H} \alpha$ image .. & $05: 04-05: 51$ & $30 \mathrm{~s}$ \\
\hline $\mathrm{H} \alpha$ (NAOC) image .. & $04: 37-05: 48$ & $70-80 \mathrm{~s}$ \\
\hline TRACE $171 \AA$ Amage & 04:54:02-05:39:01 & $40 \mathrm{~s}$ \\
\hline SXR image (Be119). & $04: 53: 31-05: 12: 27$ & $12-16 \mathrm{~s}$ \\
\hline C2/LASCO image ... & 05:30-06:30 & $20-30 \mathrm{~min}$ \\
\hline C3/LASCO image ... & $06: 18-09: 42$ & $30-60 \mathrm{~min}$ \\
\hline Yohkoh HXR flux ... & $04: 53-05: 20$ & \\
\hline
\end{tabular}

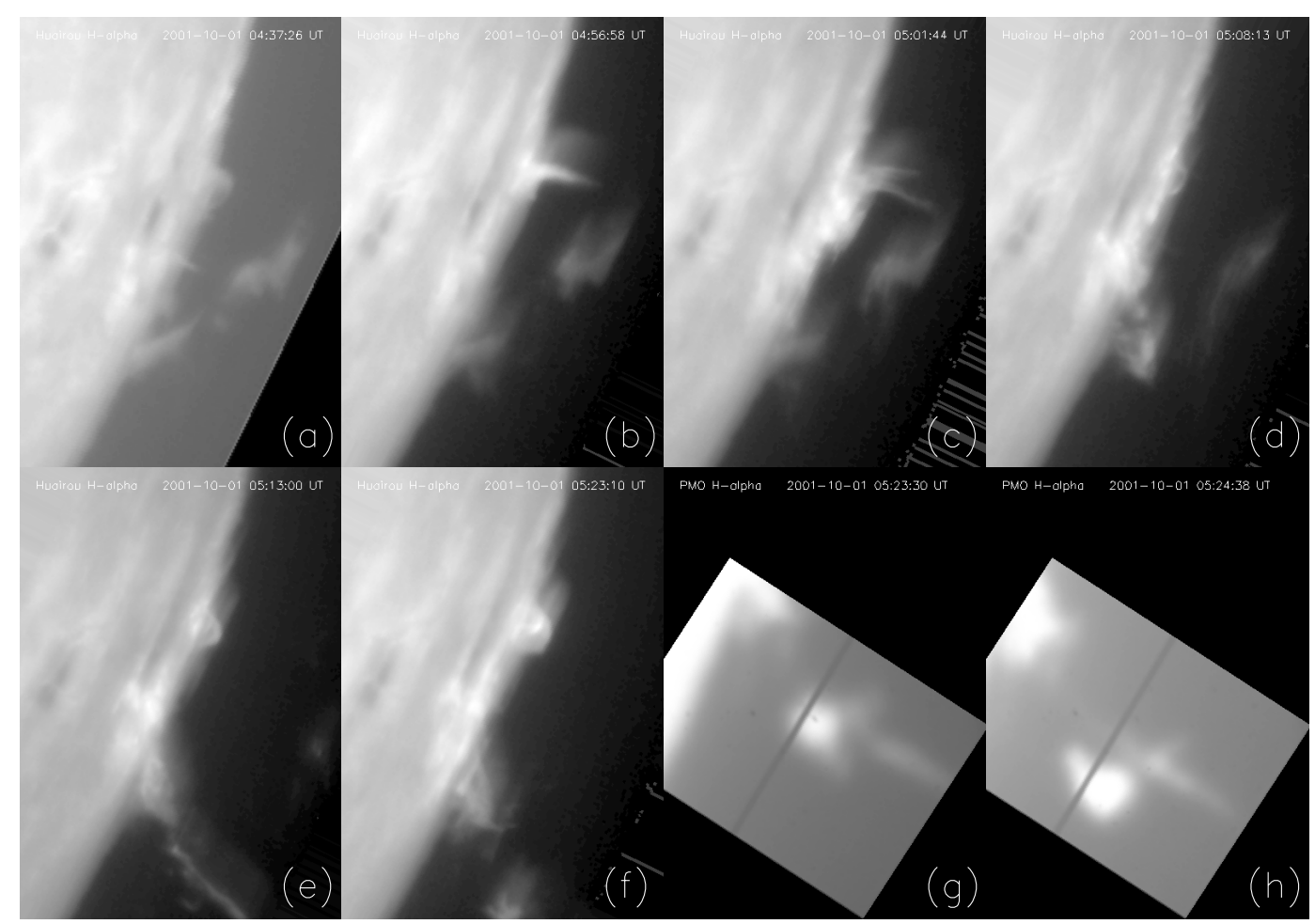

Fig. 3. H $\alpha$ images a)-f) from HSOS and g)-h) from the MISS at PMO. The observation times are 04:37:26 UT, 04:57:54 UT, 05:01:44 UT, 05:08:13 UT, 05:13:00 UT, 05:23:10 UT, 05:23:30 UT and 05:24:38 UT, respectively. The field-of-view (FOV) of the image is 200" $\times 280^{\prime \prime}$. Notice that the images in $\mathbf{g}$ )-h) are shifted to the north by about $130^{\prime \prime}$ due to the difference in observation targets. North is on the top and east to the left.

processes, and is accompanied by a CME. Even though this is not a prepared observation, and the start times and sampling rates are different at different wavebands, the observed data are still comparable. We list the observation periods and average sampling rates of the observations in different wavebands in Table 1 .

\subsection{Ho observation}

We have $\mathrm{H} \alpha$ images from the MISS at PMO and HSOS. In Fig. 3 we show mainly the $\mathrm{H} \alpha$ images from HSOS due to the limited FOV of the $\mathrm{H} \alpha$ images from the MISS. On the other hand, the HSOS $\mathrm{H} \alpha$ images have small FOV over the limb, therefore, we display $\mathrm{H} \alpha$ images from the MISS in Fig. $3 g$ and $3 \mathrm{~h}$ when the spray went higher above the solar limb. For the same reason, the mosaicked $\mathrm{H} \alpha$ image in Fig. 6 is from the MISS. From Fig. 3 we notice that there was no precursor of eruption in AR 9628 at 04:37:26 UT but an active prominence at the south-west limb. At 04:57:54 UT, there was a bright surge-like eruption at the north part of AR 9628 and its footpoint was also bright. Our $\mathrm{H} \alpha$ spectra imply that there are flare knots inside the limb, which temporally corresponds to the first peak of HXR light curves (Fig. 2) of M1 and M2 energy channels (23 keV-53 keV).

The surge darkened at 05:01:44 UT and separated into two parts. In addition, there were 2-3 lower bright arc structures to the south of the surge, which appeared to be flare loops. This is confirmed by the $\mathrm{H} \alpha$ spectra. These structures temporally correspond to the second peak of the HXR light curves (Fig. 2) and the terrace of GOES X-ray flux curve (Fig. 1) between the two peaks. However, the flare loops lasted only about $2 \mathrm{~min}$. Then the structures changed and darkened, and apparent 


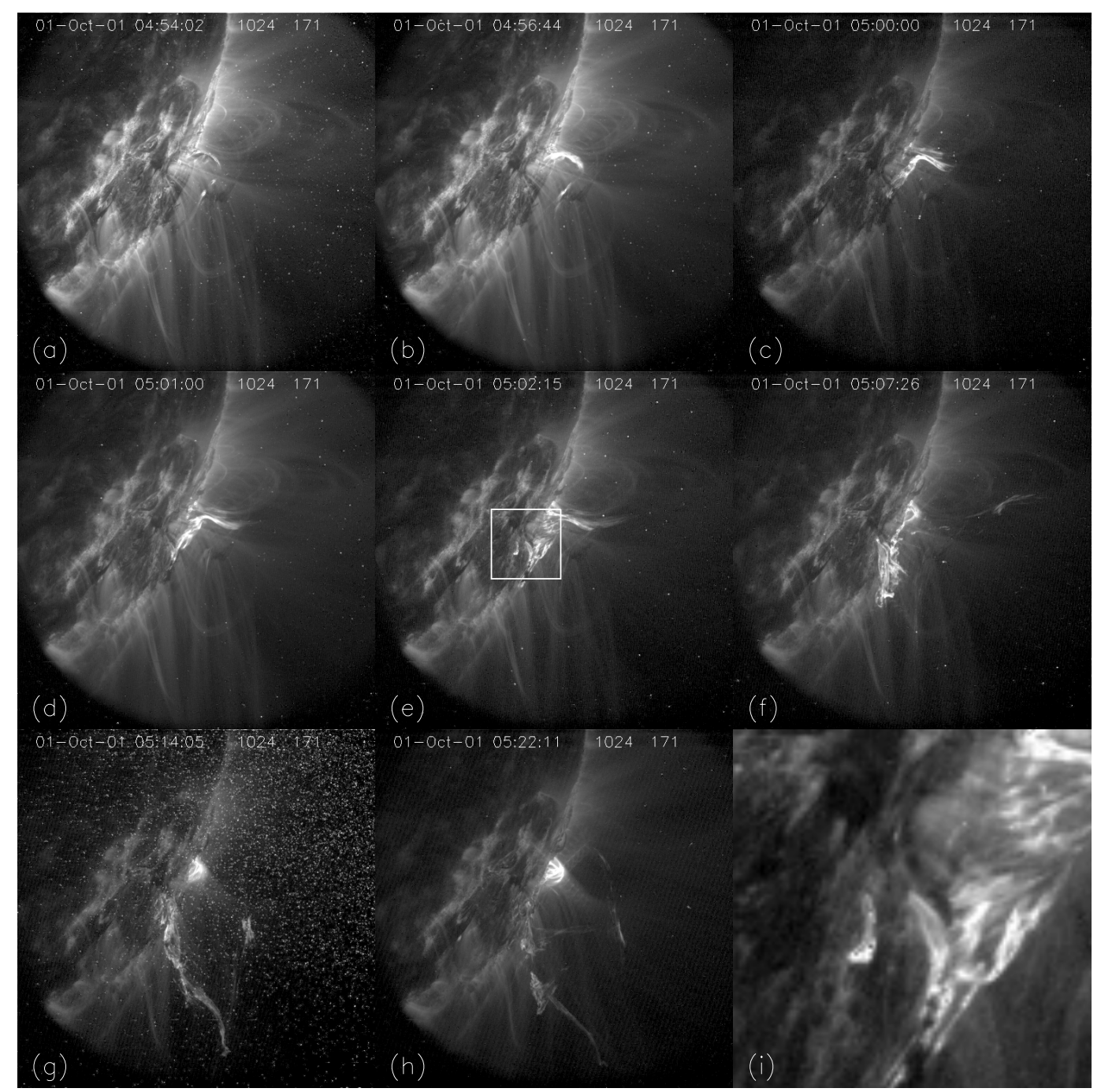

Fig. 4. EUV images at $171 \AA$ from TRACE. The observation times are 04:54:02 UT, 04:56:4 UT, 05:00:00 UT, 05:01:02 UT, 05:02:15 UT, 05:07:26 UT, 05:14:05 UT and 05:22:11 UT for a)-h), respectively. The FOV of the images is 512" $\times 512^{\prime \prime}$ for a)-h) and 100" $\times 100^{\prime \prime}$ for i) that is the enlarged image of the squared area in e) to show the loops more clearly. North is on the top and east to the left.

post-flare loops were developed. At 05:09:30 UT, there was a post-flare-loop-like hump at the edge of the surge-originated area, which lasted a long time and remained unchanged, and temporally corresponds to the main HXR peak (Fig 2).

There was a precursor of spray at 05:06:00 UT at the southern part of the active region and the large sunspot, and the spray erupted soon afterwards. The spray reached about $1.45 \times 10^{4} \mathrm{~km}$ at 05:08:13 UT. It is inferred from the $\mathrm{H} \alpha$ spectra and images that the spray erupted with high speed at 05:06:34 UT. The spray reached a height of $1.68 \times 10^{5} \mathrm{~km}$ above the limb at 05:18:30 UT and seemed to stop rising. Its front part displayed a south-bent arc structure. And soon a mass bundle, which was motionless relative to the spray, started suddenly to move outwards (Figs. $3 \mathrm{~g}$ and $3 \mathrm{~h}$ ).

\subsection{EUV observation}

The TRACE $171 \AA$ filter has a passband of $6.4 \AA$ and covers a temperature range of $1.6 \times 10^{5} \mathrm{~K}$ to $2.00 \times 10^{6} \mathrm{~K}$ with center at $1.0 \times 10^{6} \mathrm{~K}$. We also refer to the $195 \AA$ images from EIT/SOHO in our analysis because TRACE observed the Sun at $171 \AA$ only in the period of interest. We found that the images at $171 \AA$ and $195 \AA$ are quite similar. Therefore, the following descriptions and results are based on TRACE $171 \AA$ images due to its better spatial resolution $\left(\approx 1^{\prime \prime}\right)$.

Shown in Fig. 4 are $171 \AA$ images from TRACE. From this figure we see that the eruption process in EUV is morphologically similar to that in $\mathrm{H} \alpha$, but there also exist some differences. On the $171 \AA$ images, there was a south-bent arc that corresponded to the $\mathrm{H} \alpha$ surge at the north part of the active region. The arc was obviously brighter at 04:56:44 UT than two minutes before, and several weak loops developed around its footpoint. It is quite possible that a process of magnetic field opening and closing occurred during the two minutes. It is temporally consistent with the $\mathrm{H} \alpha$ surge, flare and first peak of HXR flux (Fig. 2) within the measurement accuracy.

The upper part of the bright arc changed to slightly northbent at 05:00:00 UT and some bright patches appeared south of 


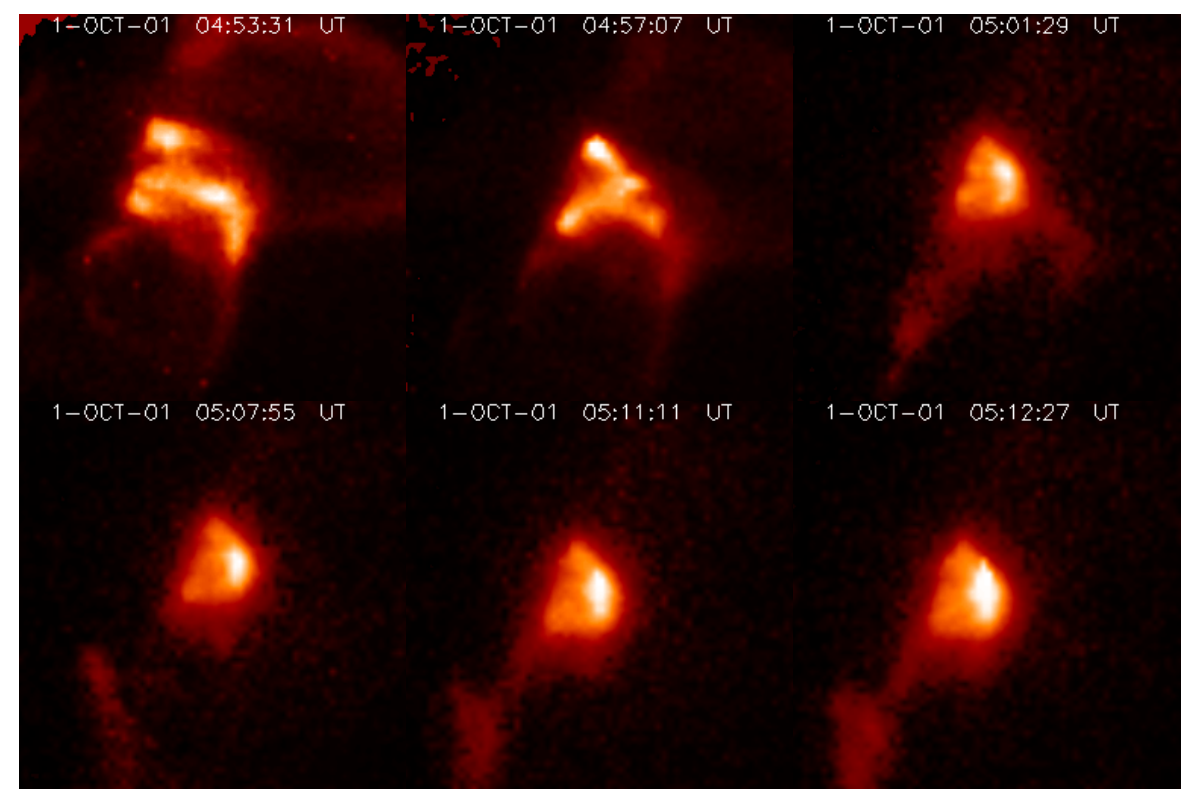

Fig. 5. SXR images from SXT/Yohkoh taken with the Be119 filter. The FOV of the images is $157^{\prime \prime} \times 157^{\prime \prime}$. The observation times are indicated on each image. North is on the top and east to the left.

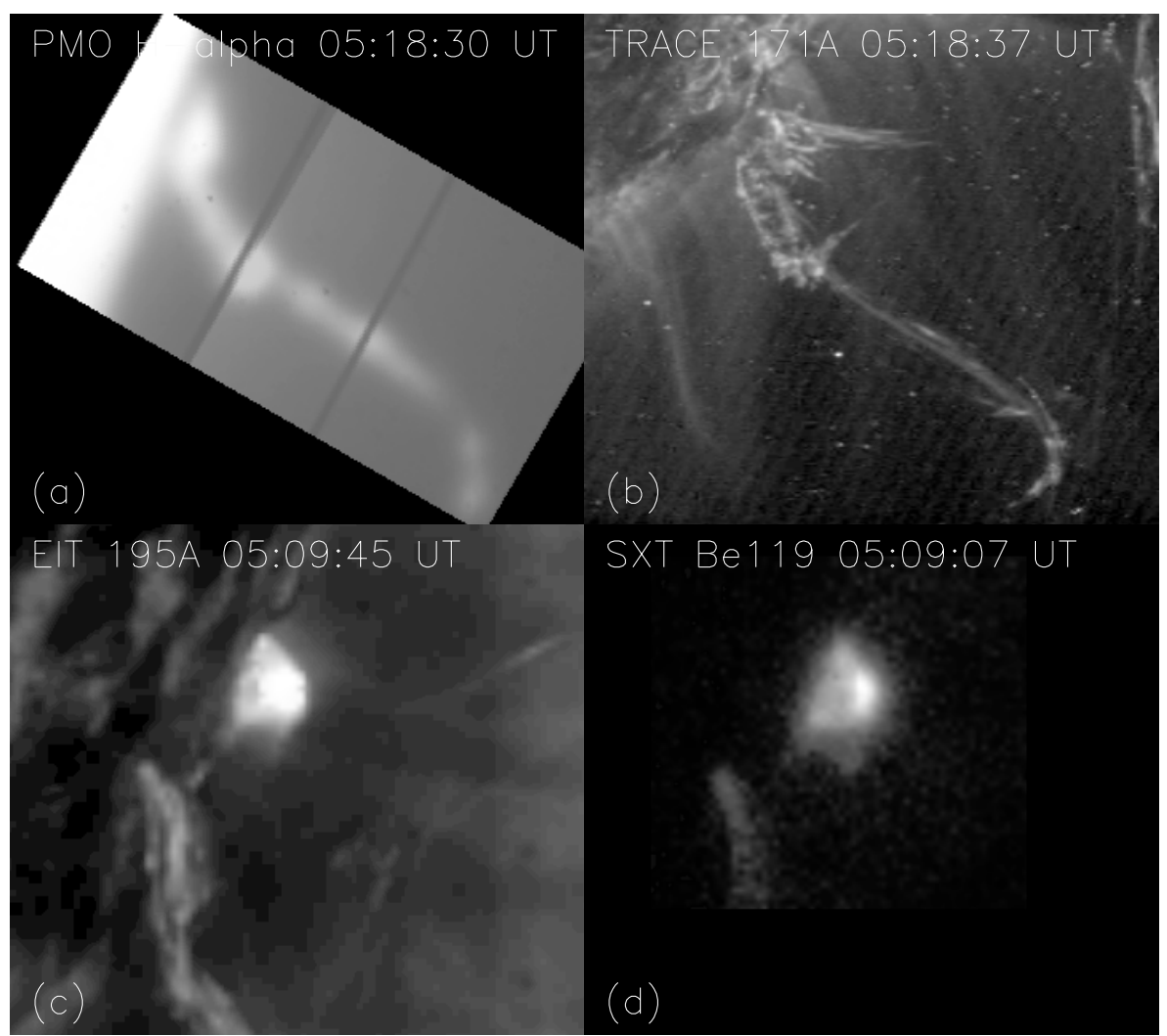

Fig. 6. a) Mosaicked H $\alpha$ image from the MISS at PMO; b) TRACE $171 \AA$ image; c) EIT/SOHO $195 \AA$ image and d) SXR image from SXT/Yohkoh with Be119 filter. The observation times are shown on each image. The FOV of the images is $240^{\prime \prime} \times 210^{\prime \prime}$. Notice that c)-d) are shifted to the north by about $80^{\prime \prime}$ with respect to a)-b) due to satellite pointing. North is on the top and east to the left.

the arc. These patches extended southward about $2.8 \times 10^{4} \mathrm{~km}$ in one minute. The locations of these patches appear to be that of the double ribbon below $\mathrm{H} \alpha$ flare loops. However, there is no lower bright flare loop like that in $\mathrm{H} \alpha$. Then, the graduallyformed dark post-flare loops covered these patches.
We found, in the $171 \AA$ image at 05:02:15 UT, that there were two thin and bright arcs connected the southern footpoint of the former double-ribbon flare with a small area east of the flare on the disk (Figs. 4e and 4i). The arcs lasted about 4 min. At 05:07:26 UT, a dramatic flare spray blew out from the 


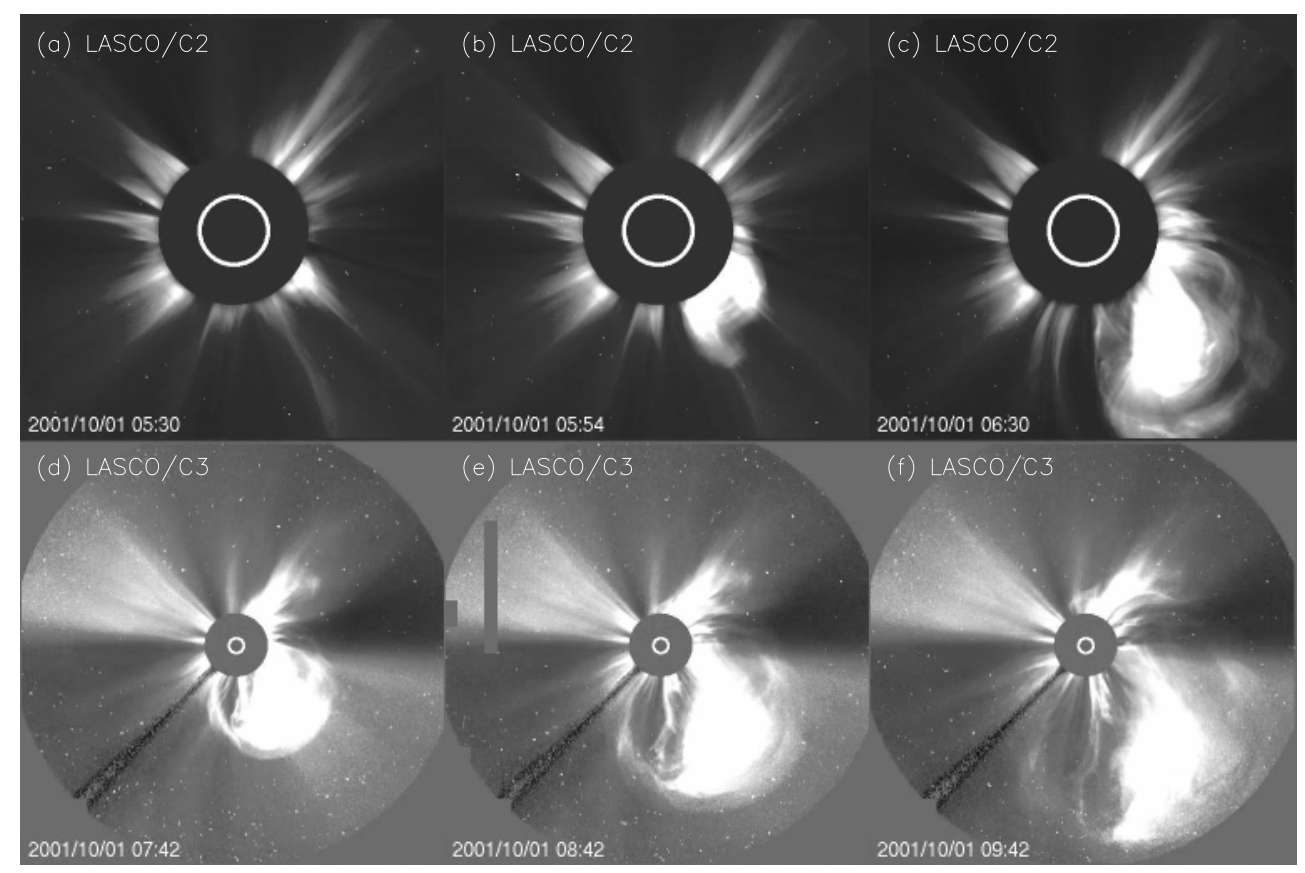

Fig. 7. a)-c) Images from C2/LASCO coronagraph and d)-e) images from C3/LASCO coronagraph on SOHO. The observation times are indicated on each image. North is on the top and east to the left.

disk-center-end of the arcs and moved over the solar limb. It is possible that the mass and energy of the spray are transported from the footpoints of the flare. We also found that the spray was twisted in its ejecting process, which is similar to and clearer than that in $\mathrm{H} \alpha$ due to the higher spatial resolutions of the $171 \AA$ images.

As in $\mathrm{H} \alpha$, the spray did not rise any more after it reached a height of $1.8 \times 10^{5} \mathrm{~km}$ and its front part was bent. Meanwhile, some mass blasted out of the FOV of TRACE (Fig. 4). It is interesting that the three distinct eruptions, originating from different locations and at different times, moved out at the same speed of $296 \mathrm{~km} \mathrm{~s}^{-1}$ in the sky plane in the first several minutes (Fig. 8a).

The loop system in the north part of the active region formed after the eruptions changed from high weak complex loops to low bright simple loops from 05:04:37 UT to 05:07:26 UT. Then the loop system heightened and became more and more complicated. It presumably resulted from multiple magnetic field reconnections. The loop system was always bright and its brightness was uniform, which is different from usual $\mathrm{H} \alpha$ and SXR loops, and has no obvious bright loop tops. The $\mathrm{H} \alpha$ post-flare loop corresponding to this EUV loop remained unchanged and weak for a long time. At 05:22 UT its top brightened suddenly, and weakened 3 min later (Fig. 3). Such a large temporal difference in flares is seldom observed. It may be related to the mass ejection in the spray, like the flare plasma that intrudes into the penumbra of a large sunspot with low X-ray flux may associate with sprays (Gallagher et al. 2000).

In addition, the ejection in the north part changed its shape while rising and weakened at 05:11:46 UT. Its upper part was bent to the south and met the second spray. We believe that all these come from the constraints of magnetic fields over the active region.

\subsection{SXR images}

Figure 5 shows SXR images from SXT/Yohkoh taken with the Be119 filter. The SXR images imply that there was loop interaction between 04:53:31 UT and 04:57:07 UT, which consequently resulted in the change of shape and brightness of the loops. The loop interaction probably leads to the first $\mathrm{H} \alpha$ flare and HXR peak (Fig. 2). On the image at 05:01:24 UT, there was a lower bright arc connecting the former two bright footpoints indicating that a reconnection happened. It temporally corresponds to the second $\mathrm{H} \alpha$ flare and the second eruption. Meanwhile, we noticed that south of the arc weak loops formed and moved southward, which is consistent with the $\mathrm{H} \alpha$ and EUV flare loops.

It can be seen in the SXR image at 05:07:55 UT that there was a newly-born and clear mass ejection from the southern part of the active region. Comparison with $\mathrm{H} \alpha$ and EUV images indicates that its location is the same as the large $\mathrm{H} \alpha$ and EUV spray (Fig. 6). It is of great interest that the spray showed almost the same shapes in $\mathrm{H} \alpha$, EUV and SXR, which are formed in plasmas with temperatures that differ by a factor of more than $100\left(1.0 \times 10^{4} \mathrm{~K}-1.3 \times 10^{6} \mathrm{~K}\right)$, and kept relatively stable for a time. Its ejecting trajectory is undoubtedly confined by the magnetic fields above the region. The SXR image at 05:12:27 UT has the largest bright loop-top area and brightness. This corresponds to the main peak of GOES X-ray flux of $0.5-4 \AA$ but about $3 \mathrm{~min}$ earlier than that of $1-8 \AA$. The 1-8 $\AA$ X-ray flux reflects the thermal process more than the 0.5-4 $\AA \mathrm{X}$-ray flux. However, we could not distinguish 

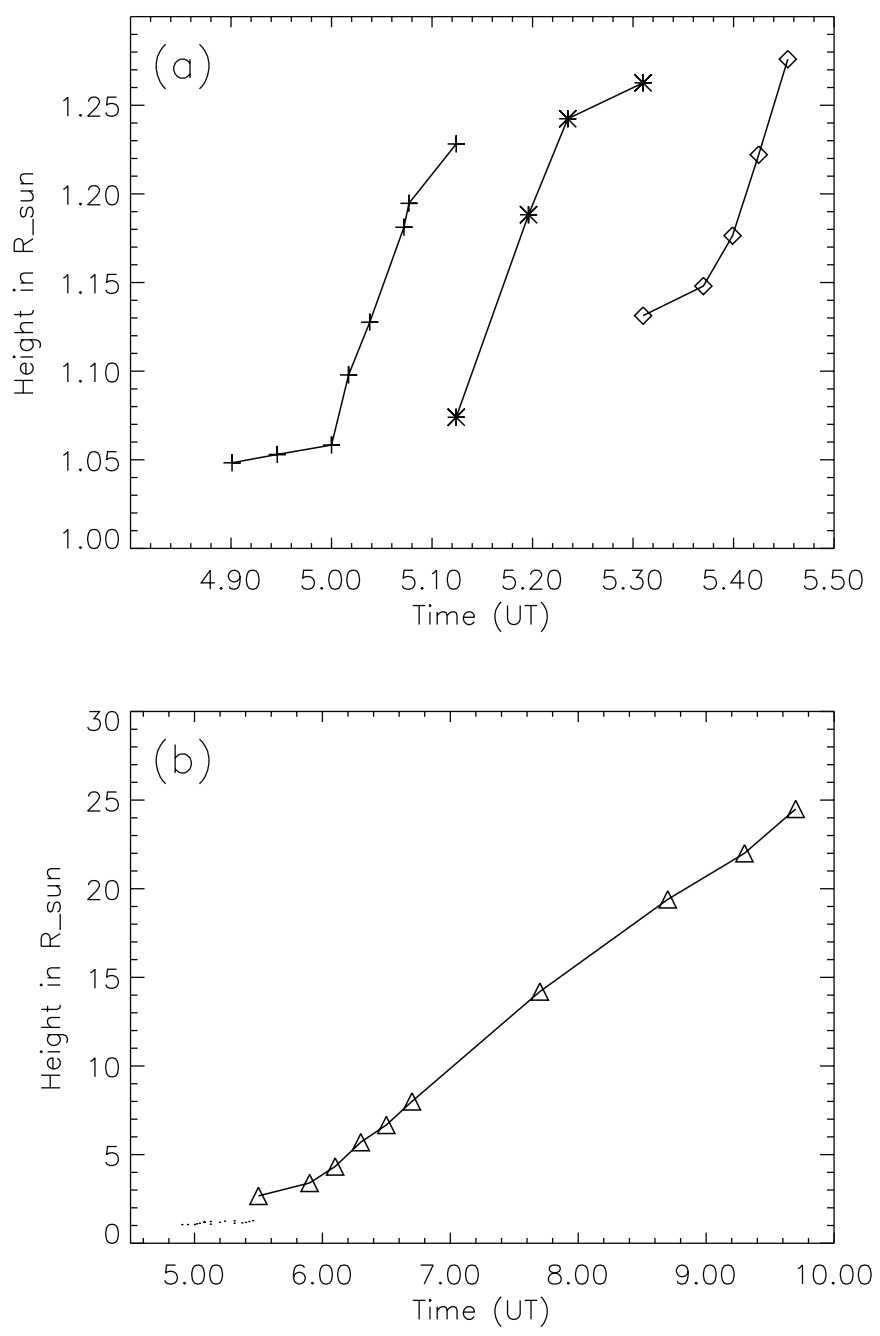

Fig. 8. The height-time curves of a) the three distinct ejections, and b) the CME. Also plotted in b) by dots (.) are the curves in a) for comparison.

any fine structure from the SXR images due to the limited spatial resolution. It is quite possible that multiple reconnections led to the large bright area on the loop top and the series of small peaks on the HXR light curves in this period (Fig. 2).

\subsection{The corona}

Shown in Fig. 7 are some images from the C2 and C3/LASCO coronagraphs on SOHO. From the images taken by $\mathrm{C} 2$ at 05:30 UT we can see there was a CME between the azimuthal angles $238^{\circ}-242^{\circ}$ (measured counterclockwise from the north). Its trajectory was not radial but bent to the south. On the image taken by C3 at 09:42 UT, the leading edge of the CME front was almost at the south direction. The largest angular width of the CME subtending the solar disk center is greater than $70^{\circ}$. The foot part of the CME always anchored in an unchanged area on the disk during the whole CME process.

An examination of the daily LASCO movies shows that this spectacular event does show evidence of the classical "3-part" structure of CMEs. The bright core part goes almost directly south and is surrounded by an elongated void region (Figs. 7d,f) in the late phase. The brightest part of this asymmetrical CME extends to the southwest of the void. Flow from this eruption continues throughout the entire day.

Gopalswamy et al. (2000) pointed out that the propagation of CME sometimes may be non-radial. For example, during a solar minimum period, CME frequently goes non-radially in the initial phase and then radially in the late phase. The behavior of the observed CME here is just the opposite. It moves almost along the radial direction in the early phase, and then quickly bends to the south and shows an asymmetrical structure. It is well known that the propagation of CME is significantly affected by the large-scale structure of the solar magnetic fields (Gopalswamy et al. 2000). Therefore, the non-radial propagation trajectory of this CME may reflect some information about the structure of magnetic fields in the middle and outer corona.

We measure the height of the CME at the leading edge at the azimuthal angle of $230^{\circ}$. Our measurement gives a rather high speed of $1089 \mathrm{~km} \mathrm{~s}^{-1}$ in the sky plane for the CME (Fig. 8b). We can infer that the CME is closely associated with the flare spray after comparing the linear part of the heighttime curve of the CME and the height-time curve of the flare spray and taking into account that they are ejected in the same azimuthal direction. We will discuss this point in detail in the next section. The radio-frequency spectrograms $(2.6-3.8 \mathrm{GHz}$, $5.2-7.6 \mathrm{GHz}$ ) from NAOC did not show any type II burst while the SGD data imply that in the metric waveband $(57-220 \mathrm{MHz})$ there were two type III bursts in the period from 05:22 UT to $05: 25 \mathrm{UT}$.

After combining the above-mentioned results and the height-time curves of the flare spray and the CME in Fig. 8, we can infer the following conclusion: the CME was accelerated at about $2 R_{\odot}$ and the large-scale coronal magnetic field changed its structure.

\subsection{Spectra}

Presented in Fig. 9 are spectrograms in H $\alpha$ (left), Ca II $8542 \AA$ (middle) and He I $10830 \AA$ (right) taken by the MISS in the initial (upper) and later (bottom) phase of the flare spray. The figure shows that the spectral profiles are unusually broadened and largely blue-shifted with a maximum velocity of $286 \mathrm{~km} \mathrm{~s}^{-1}$ in the initial phase of the large spray. The initial ejecting velocity of the CME is as high as $412 \mathrm{~km} \mathrm{~s}^{-1}$ when taking into account both the velocity along line-of-sight and that in the sky plane.

The line-of-sight velocity of the spray decreased significantly (Fig. 9, lower row) during the ejecting process while its speed in the sky plane only slightly changed. This presumably indicates that in the spray process not only its velocity decreases but its travelling direction changes as well.

\section{Discussion and conclusion}

Our observations show that the discussed series of erupting events are initiated by the interaction of loops above the active region. The reconnections of loops above the active region have a good association with the HXR bursts. Although the high energy activities of this active region concentrated on 


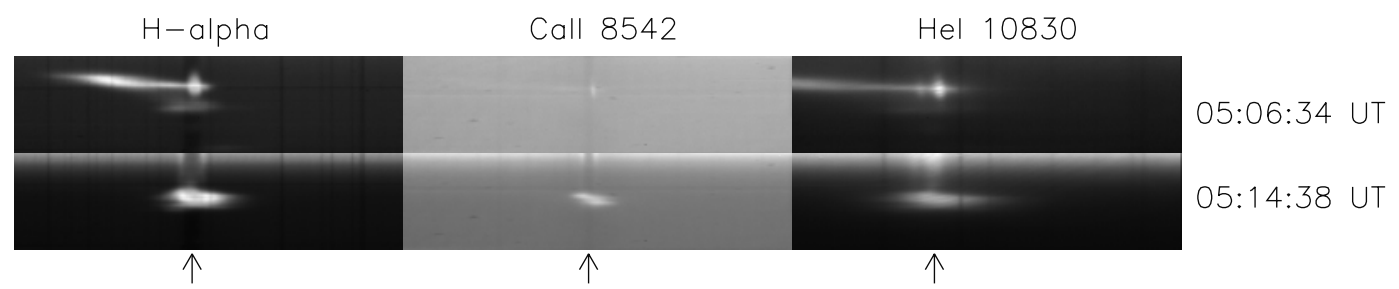

Fig. 9. Spectrograms of $\mathrm{H} \alpha$ (left), Ca II $8542 \AA$ (middle) and $\mathrm{He}_{\mathrm{I}} 10830 \AA$ A (right) taken at 05:06:34 UT (upper) and 05:14:38 UT (bottom) by the MISS at PMO. The arrows below the spectrograms refer to the centers of the emission lines.

the northern part of the region, the data and analysis suggested that both the flares in the northern and the middle part and the large spray was intimately connected to the high energy area.

We found that there are low bright arcs connecting the onset location of the large spray and footpoints of the flare loops. We observed and revealed that the CME that erupted soon afterwards is intimately associated with the flare spray, and had been accelerated at around $2 R_{\odot}$.

Since the first observation of CME, solar physicists have been searching for the relation between CME and other ejections. It was found that some CMEs are associated with erupting prominences or flares while the others not. Now the commonly accepted viewpoint is the suggestion proposed by Harrison (1995). He pointed out that flare and CME are signatures of the same magnetic "disease", i.e., they represent the responses in different parts of the magnetic structure to a particular activity; they do not drive one another but are closely related. The consistence of the onset times and azimuthal angles of the observed flare spray and CME in our case lead us to conclude that they are closely associated. Meanwhile, there are two type III bursts in the metric waveband at that time. If they are produced above the active region, indicating that there exist motions of electron beams of high energy, we may infer that they are associated with the reconstruction of the large-scale magnetic field. However, because we do not have more reliable observational data, such as images from C1/LASCO, we do not know whether the flare spray triggered the CME. On the other hand, both the flare spray and CME may be the products of a single large-scale process.

There are two interesting phenomena worth mentioning in our observations. The first is that the three distinct ejections have the same speed in the sky plane. The second is that the low and high temperature materials can exist in the same observed large spray as manifested by the $\mathrm{H} \alpha$, EUV and SXR observation above. The spray has nearly the same shape in $\mathrm{H} \alpha$, EUV and SXR. The SXR images presented in this work are taken by the SXT/Yohkoh with the Be119 filter, which has a lower temperature cut-off of $1.3 \times 10^{6} \mathrm{~K}$ (Tsuneta et al. 1991). This indicates that the plasmas with temperature differing by a factor of more than 100 can coexist and stay relatively stable in an ejection at as high as $1.8 \times 10^{5} \mathrm{~km}$. SkyLab observations showed that prominences may take roughly the same shape in spectral lines formed in the temperature range of $1.0 \times 10^{4} \mathrm{~K}-3.0 \times 10^{5} \mathrm{~K}$ (Wang 1998). Our observations provide a wider temperature range in which plasmas can co-exist and a better constraint for theoretical interpretation. So far, we have not proposed a mechanism to explain well the observed phenomenon. We will present the detailed analysis of the observed spectra in multiple wavebands and the studies of dynamical process of this event in future papers.

Acknowledgements. We would like to thank the referee for his/her valuable comments and suggestions. We are also grateful to professor B. R. Zhang for his help in preparing the data. SOHO is a project of international cooperation between ESA and NASA. TRACE is a NASA Small Explorer project. This work was supported by the National Natural Science Foundation of China (NSFC, grant number 49990451), National Basic Research Priorities Project (G2000078402) of China, and Chinese Academy of Sciences.

\section{References}

Cox, A. N. (ed.) 1999, Allen's Astrophysical Quantities, Fourth edition (Springer), 374

Brueckner, G. E., Howard, R. A., Koomen, M. J., et al. 1995, Sol. Phys., 162, 357

Delaboudinière, J.-P., Artzner, G. E., Branaud, J., et al. 1995, Sol. Phys., 162, 291

Gallagher, P. T., Williams, D. R., Phillips, K. J., et al. 2000, Sol. Phys., 195,367

Gopalswamy, N., Hanaoka, Y., \& Hudson, H. S. 2002, Adv. Space Res., 25, 1851

Handy, B. N., Acton, L. W., Kankelborg, C. C., et al. 1999, Sol. Phys., 187,229

Harrison, R. A. 1995, A\&A, 304, 585

Harrison, R. A., Sawyer, E. C., Carter, M. K., et al. 1995, Sol. Phys., 162,233

Harrison, R. A., Bryans, P., \& Bingham, R. 2001, A\&A, 379, 324

Kosugi, T., Makishima, K., Murakami, T., et al. 1991, Sol. Phys., 136, 17

Li, H., Fan, Z. Y., \& You, J. Q. 1999, Sol. Phys., 185, 69

Li, H., You, J. Q., Wu, Q. D., \& Yu, X. F. 2002, Chin. Phys. Lett., 17, 742

Maeda, K., Tameraga, T., \& Kubota, J. 1978, PASJ, 30, 533

Öhman, Y., Stiber, G., \& Kasoffsky, U. 1967, Sol. Phys., 1, 60

Tandberg-Hanssen, E., Martin, S. F., \& Hansen, R. T. 1980, Sol. Phys., 65, 357

Tsuneta, S., Acton, L., \& Bruner, M. 1991, Sol. Phys., 136, 37

Valniček, B. 1964, Bull. Astron. Inst. Czech., 15, 20

Wang, H. M. 1998, Sol. Phys., 183, 91

Warwick, J. W. 1957, ApJ, 125, 811

Webb, D. F., \& Jackson, B. V. 1981, Sol. Phys., 73, 341 